\title{
Indications de la recherche des anticorps anti-spermatozoïdes
}

\author{
Claire Barthélémy \\ Biologie de la Reproduction - CECOS, TOURS
}

\section{RESUME}

Les causes immunologiques de l'infertilité du couple sont souvent évoquées mais sont à l'heure actuelle toujours contestées. La prévalence des anticorps anti-spermatozoïdes chez l'homme varie de 2,8 à $26 \%$ et de 0,2 à $1,6 \%$ chez la femme. Les antigènes spermatiques n'apparaissant qu'à la puberté, toute pathologie mettant en contact ces antigènes avec le système immunitaire peut entraîner une immunisation. II existe des mécanismes anatomiques et physiologiques actifs pour éviter cette réponse immunitaire chez l'homme et la femme. De nombreux effets défavorables des anticorps anti-spermatozoïdes sur la fertilité ont été décrits : altérations des paramètres spermatiques, non-pénétration des spermatozoïdes dans le mucus cervical, interactions ovocyte-spermatozoïdes perturbées. Les indications de la recherche des anticorps chez l'homme et la femme sont basées sur des signes d'appel cliniques et biologiques.

Chez l'homme, tout antécédent de chirurgie urogénitale, d'obstruction congénitale ou acquise sur le tractus génital, et de pathologie du tractus notamment les infections et/ou inflammations, sont une indication de la recherche des anticorps antispermatozoïdes (ASA). Souvent les patients porteurs d'ASA n'ont pas d'antécédents pathologiques retrouvés, et seules les anomalies du spermogramme, les résultats négatifs ou "pauvres" des tests dans la glaire et les échecs de fécondation in vitro (FIV) classique, incitent à les rechercher.

Chez la femme, la pathogenèse de cette immunisation est moins claire que chez l'homme. Ont été incriminées les infections pelviennes hautes, l'endométriose, les fausses couches à répétition, les pratiques sexuelles et les interventions au niveau du col utérin. Comme chez l'homme, les tests dans la glaire sont négatifs ou déficients et on retrouve des échecs de FIV inexpliqués.

La conséquence du succès de l'ICSI dans cette indication est la diminution de la recherche sur les anticorps anti-spermatozoïdes. Avoir un diagnostic exact, une explication probable de l'infertilité du couple est indispensable car elle peut aider à la progression de la connaissance sur l'histoire naturelle de l'apparition des ces anticorps, sur l'identification des antigènes impliqués dans ce processus, et ainsi permettre le développement de tests de plus en plus spécifiques afin d'engager une stratégie de prise en charge plus rapide du couple à l'aide de la procréation médicalement assistée la plus adaptée.

Mots clés : anticorps antispermatozoïdes, ASA, infertilité immunologique, sperme humain

\section{Correspondance :}

Dr Claire Barthélémy - Biologie de la Reproduction, CECOS TOURS, CHU Bretonneau, 2 Bis Boulevard Tonnellé, 37044 Tours cedex - Tel 02.47.47.84.94 - Fax 02.47.47.84.84 Email claire.barthelemy@chu-tours.fr 


\section{INTRODUCTION}

Depuis plus d'un siècle on sait que les animaux sont capables de développer une auto-immunisation contre les spermatozoïdes (Landsteiner), mais c'est depuis les travaux de Rümke en 1954 que l'on connaît leur impact sur la fertilité chez l'homme [89]. L'homme et la femme sont susceptibles de fabriquer des anticorps contre les spermatozoïdes humains. Chez l'homme, il s'agit d'une auto-immunisation, chez la femme d'une iso-immunisation. Ces anticorps sont de la classe des IgG, et/ou des IgA (plus particulièrement sécrétoires au niveau du tractus génital) et/ou des IgM retrouvées plus dans le sérum que dans les sécrétions génitales [61, 103]. Ils peuvent être agglutinants, cytotoxiques ou immobilisants [75]. Ces états pathologiques, le plus souvent idiopathiques, sont susceptibles d'altérer la fertilité du couple et entraînent une diminution des grossesses spontanées avec un allongement du délai à concevoir [1]

Cette immunisation est rare chez les couples fertiles (moins de 1\%). La prévalence des anticorps chez l'homme infécond varie de 2,8 à 26\% selon les études en comparaison des $2,5 \%$ retrouvés dans une population d'hommes fertiles $[25,45,72,83]$. Cette immunisation est plus importante chez les hommes que les femmes: on ne retrouve que $0,2 \%$ à $1,6 \%$ d'anticorps chez la femme infertile $[14,18$, $30,61]$. Il a été décrit une corrélation significative entre la positivité chez la femme et l'homme [105]. Les variations importantes de ces fréquences sont expliquées par les critères de sélection des populations étudiées, la nature et la spécificité des techniques utilisées, le type d'anticorps recherché, les seuils de positivité retenus et les compartiments testés, à savoir, sérum, mucus, sperme et spermatozoïdes [83].

Les causes immunologiques de l'infertilité du couple sont souvent évoquées mais sont à l'heure actuelle toujours débattues $[2,36,48,68,69]$.

\section{PHYSIOPATHOLOGIE}

Chez l'homme, la tolérance aux auto-antigènes se développe tôt dans la vie fotale alors que les cellules spermatiques ne se forment qu'à la puberté. L'auto-immunisation s'expliquerait donc par le concept d'antigène exclu (théorie de Burnet). Ces antigènes spécifiques produits à la puberté, au cours de la spermatogenèse et de la maturation du gamète, ne seraient pas reconnus comme les siens par le système immunitaire entraînant une réaction immunitaire prévisible. Il existe des mécanismes anatomiques et physiologiques actifs pour éviter cette réponse immunitaire chez l'homme et la femme.

\section{Chez l'homme}

La barrière hémato-testiculaire très sélective créée, entre autres, par les jonctions serrées des cellules de Sertoli isole les spermatocytes post-méiotiques du tissu interstitiel à partir du stade pachytène, limitant l'extravasation et la phagocytose par les cellules immunocompétentes. Toute rupture de cette barrière entraîne le passage de cellules immunocompétentes vers le tractus et/ou la transsudation $d$ 'antigènes spermatiques vers le tissu interstitiel. Il se produit rapidement une réponse immunitaire objectivée par une réaction inflammatoire locale suivie de la production d'anticorps anti-spermatozoïdes [26]. Cette barrière a une action limitée car le compartiment basal n'est pas protégé et les cellules pré-leptoténes sont elles aussi antigéniques. Un passage est aussi toujours possible à partir du rete testis et des cônes efférents, même si la barrière endothéliale joue un rôle régulateur sur la pénétration de cellules du système immunitaire ou le relargage d'antigènes spermatiques dans la circulation générale. La fixation des anticorps sur le gamète se situe probablement dans tout le tractus puisque la maturation des spermatozoïdes, avec une modification des antigènes de surface, est continue tout au long de l'appareil génital, y compris au contact du plasma séminal [74].

D'autres mécanismes immunomodulateurs complètent cette immunotolérance $[33,42,103]$. Il existe des mécanismes d'immunorégulation locale intra-testiculaire : production de molécules immunosuppressives par la cellule Sertoli (TNF $\alpha$, IL6) et le système Fas/FasL système qui régulerait l'homéostasie immunologique dans les conditions physiologiques ou pathologiques, en induisant l'apoptose des lymphocytes les plus agressifs [33, 42]. On évoque chez le rat le rôle immunosuppresseur dans le testicule de certains macrophages qui bloqueraient la réaction inflammatoire [42].

Il existe une relation inverse entre les cellules $T$ supresseur et la présence d'ASA [53]. Les cellules lymphoïdes T (suppresseur/cytotoxique) sont présentes majoritairement dans l'épithélium du rete testis, du déférent, de la prostate et du plasma séminal, et sont activées sélectivement, notamment par des activateurs solubles dans le sperme ou présents sur la surface des gamètes, alors que les cellules $\mathrm{T}$ helper sont plutôt localisées dans le tissu interstitiel [7]. Les spermatozoïdes et le plasma séminal peuvent ainsi initier une immunosuppression [53]. La production d'ASA pourrait être la conséquence d'une diminution de l'activité ou de la quantité des cellules $\mathrm{T}$ et/ou le déclin ou absence des facteurs qui recrutent et activent ces cellules. Il est possible aussi qu'une antigénicité spermatique altérée entraîne une suppression inadéquate de la réponse immune aux spermatozoïdes [53, 103].

Les taux du complément sont très bas dans l'épididyme, et le plasma séminal contient des protéines anti-complémentaires qui vont croissant au cours de la spermatogenèse, et 
s'attachent sur le spermatozoïde au cours de leur sécrétion épididymaire. La formation d'ASA cytotoxiques est rare si ce mécanisme physiologique n'est pas perturbé $[22,87]$.

\section{Chez la femme}

L'étiologie de cette iso-immunisation apparaît moins claire. Durant la vie génitale, le tractus est inséminé de façon répétée par les spermatozoïdes, et seule une minorité de femmes développent des anticorps [70]. L'épithélium vaginal plus épais retarde effectivement le passage des gamètes dans le système vasculaire sous-jacent [72]. Les effractions muqueuses et les infections génitales, qui mettront ces antigènes spermatiques en contact avec les cellules immunocompétentes, sont susceptibles d'entraîner une production locale ou systémique d'anticorps [45]. Une immunisation croisée est toujours possible avec les antigènes bactériens ou viraux notamment dans les problèmes infectieux.

L'activation de ces cellules $T$ suppresseur dans le cervix après un rapport limite la réponse immune aux spermatozoïdes. La présence de protéines anti-complémentaires locales et séminales limitera la production d'anticorps cytotoxiques. La présence d'anticorps chez la femme peut donc être la conséquence, soit d'une déficience de recrutement des cellules $T$ supresseur du cervix et/ou de leur incapacité à être activées par les activateurs du plasma séminal, soit de l'induction d'une réponse immune due aux facteurs activateurs des $\mathrm{T}$ helper du plasma séminal [12, 67, 103].

\section{INTERFERENCE DES ASA AVEC LA FERTILITE}

De nombreuses études contradictoires existent quant à l'importance clinique des anticorps dans l'infertilité masculine [18]. Par contre, il semble que la présence d'ASA même à un taux bas dans le mucus cervical soit une cause sévère d'infertilité féminine [30]. Il existe des arguments cliniques et biologiques importants en faveur de leur effet sur la fertilité $[18,36,70,83,103,104]$. Ils constituent souvent le seul facteur étiologique retrouvé [48]. Ces anticorps entraînent des perturbations à plusieurs niveaux :

\section{Anomalies du spermogramme}

Elles sont constituées par l'agglutination des spermatozoïdes, la diminution de la mobilité progressive, de la vitalité et quelquefois de la numération des spermatozoïdes $[8,25$, $26,110]$.

\section{Interférence avec la pénétration des spermatozoï- des dans le mucus cervical}

Cette pénétration est faible ou nulle, avec immobilisation et/ou mobilité anormale des spermatozoïdes ; ces anomalies résultent en une perturbation des tests de compatibilité glaire / sperme in vivo et in vitro [29, 30].

\section{Effets sur les interactions ovocyte-spermatozoïdes}

Les anticorps dirigés contre le gamète dans sa totalité ou contre des antigènes particuliers peuvent interférer avec les fonctions spermatiques impliquées dans la fécondation [92, 109]. Ces effets ont été mis en évidence par des études rétrospectives et prospectives sur la fécondation in vitro (FIV). Ces effets sont discutés car certains auteurs ne retrouvent pas de différence en terme de grossesses malgré un pourcentage de fécondation diminué $[88,98]$. La corrélation négative avec le taux de grossesses en FIV est fonction du pourcentage et de la localisation des anticorps liés aux gamètes (>90\%), et de leur type $[1,16,67,110,111]$. Cette altération intervient à plusieurs niveaux du processus de fécondation :

- perturbation de la réaction acrosomique par rupture prématurée de l'acrososme et/ou par blocage de la capacitation $[6,35,41,110]$;

- diminution de l'attachement à, et de la pénétration dans la zone pellucide entraînant une diminution du pourcentage de fécondation $[1,41,65,99,107]$. Une étude récente suggère que ce n'est pas l'attachement à la zone pellucide qui serait modifié mais plutôt l'induction de la réaction acrosomique par celle-ci [35] ;

- la fusion avec l'ovocyte serait empêchée par des anticorps dirigés contre les antigènes de la membrane interne de l'acrosome [65].

\section{Effets post fécondation}

Une altération du développement embryonnaire précoce a été décrite : le pourcentage de clivage diminue avec les ASA ; ceci est du à la présence d'anticorps contre la protéine de clivage CS1 [79]. Toutefois les résultats récents de l'ICSI semblent contredire ces études, même si la qualité embryonnaire est médiocre $[15,17,77]$. Enfin, des anticorps bloquants les isoantigénes sur la membrane de l'œuf pourraient contrarier l'implantation [99, 103].

\section{INDICATIONS DE LA RECHERCHE DES ASA CHEZ L'HOMME}

La stérilité d'origine immunologique est incriminée dans de nombreuses situations : il existe des signes d'appel cliniques et biologiques qui incitent à la recherche d'une auto-immunisation.

\section{Signes d'appel cliniques}

De nombreuses études évoquent les différents antécédents cliniques incriminés ; elles sont souvent conflictuelles, ce qui n'est pas étonnant car elles différent toutes par la méthodologie et les paramètres étudiés $[43,56,66,68,72]$. Les antécédents évocateurs sont les suivants :

\section{a) Chirurgie urogénitale :}

Il est reconnu que l'apparition d'anticorps est corrélée à la 
présence d'anomalies génitales chez l'enfant, indépendamment d'une chirurgie [90]. La cryptorchidie, l'ectopie uni ou bilatérale sont les plus fréquemment citées avec $66 \%$ d'ASA versus $2,6 \%$ chez les hommes fertiles pour Urry et coll. [97]. Le risque d'apparition des ASA est majoré à la puberté avec 38\% versus $11,1 \%$ chez l'enfant $[9,90]$. L'intervention chirurgicale chez les enfants prépubéres ne génère pas d'ASA, même deux ans après, alors qu'ensuite ils apparaissent chez l'adulte : $39 \%$ pour Mirilas et coll. [76]. L'âge de l'orchidopexie est un facteur significatif dans l'apparition ultérieure des anticorps avec 14,2 ans versus 8,6 ans [97]. Une étude récente de Nistal et coll. en 2002 retrouve dans les testicules de $44 \%$ des patients porteurs d'une cryptorchidie unilatérale des infiltrats inflammatoires qui pourraient être responsable de l'auto-immunisation [82]. Même si les anticorps ne sont pas la cause majeure des anomalies spermiologiques retrouvées dans les cryptorchidies, c'est toujours un facteur important à prendre en compte [97].

Une cure de hernie inguinale dans l'enfance peut entraîner une obstruction défèrentielle et la présence d'ASA dans $54 \%$ des cas à l'âge adulte [71] mais pas en prépubère [76].

La vasectomie est un bon modèle expérimental, et on a maintes fois prouvé chez l'animal l'effet de la vasectomie sur la production d'anticorps [100]. En effet, près de la moitié des hommes qui ont une vasectomie développent des anticorps. Selon les études, les taux varient de 34 à $74 \%[56,57]$, avec un pic 6 à 12 mois après l'intervention. On peut en retrouver jusqu'à 20 ans après l'intervention. Il y a plus d'anticorps circulants que d'anticorps séminaux après vasectomie, et c'est l'inverse après vaso-vasostomie. La présence de ces anticorps et leurs isotypes influent grandement sur la fertilité ultérieure après vaso-vasostomie. Bien que l'on ne puisse pas prédire la qualité du sperme après vaso-vasostomie, ceux qui ont un taux d'anticorps élevés au départ ont une fertilité nettement compromise surtout en présence d' IgA [73].

L'auto-immunisation, décrite par Hjort et coll. en 1974, survenant après biopsies testiculaires [47], n'a pas été confirmée dans les publications récentes, dans le sérum de patients ayant bénéficié de biopsies à l'aiguille ou à ciel ouvert [93].

Il n'y pas de majoration du taux d'ASA dans le sérum après anastomoses épididymo-défèrentielle, mais il préférable, pour Kay et coll., de tester les ASA avant d'envisager l'intervention car en présence d'ASA il n'a pas observé de grossesse spontanée dans son étude [59].

\section{b) Obstruction sur le tractus :}

On retrouve des ASA dans les azoospermies obstructives congénitales ou acquises, quelles soient uni ou bilatérales, que l'obstacle soit épididymaire ou déférentiel $[9,71,73$, 4]. La pathogenèse de cette immunisation a été bien décrite chez le rat. L'obstruction peut entraîner une distension des canaux et une augmentation de la pression intraluminale avec extravasation du sperme dans le tissu interstitiel et formation de granulome. Les anticorps formés au cours de ce processus reconnaissent des antigènes différents en fonction du niveau de l'obstruction, conséquence des changements maturationnels des spermatozoïdes au cours de leur transit [34].

Dans les obstructions congénitales, la plus décrite est l'agénésie congénitale bilatérale des canaux déférents (CBAVD). Il n'est donc pas surprenant de trouver aussi des anticorps chez les patients atteints de mucoviscidose porteurs de cette anomalie $[9,23]$. On retrouve pour $29 \%$ des cas des ASA dans le sérum, pour $16 \%$ dans le fluide épididymaire et pour $35 \%$ sur les gamètes [86]. Ces anticorps apparaissent en général après la puberté [9]. La longueur de l'épididyme est déterminante pour la fréquence des ASA. S'il n'y a qu'un petit résidu de la tête (au contraire d'une obstruction distale), il n'y a pas d'anticorps, ce qui montre le rôle important de la maturation épididymaire dans la génération des anticorps pour De Kretser et coll. [28].

\section{c) Autres antécédents médicaux :}

La torsion testiculaire, causant une rupture de la barrière hémato-testiculaire à cause de l'ischémie, serait génératrice d'ASA mais n'aurait que peu d'effets avant la puberté pour certains auteurs [37]. Une auto-immunisation a toutefois été décrite chez l'animal [62] et chez l'homme en période pré et post-pubertaire [90].

La varicocèle, dont l'implication dans la fertilité a fait couler beaucoup d'encre, serait potentiellement vecteur d'auto-immunité. La cause en serait la stagnation sanguine dans le plexus pampiniforme, entraînant un drainage veineux anormal du testicule et des anomalies épididymaires. Des études montrent un nombre significatif de patients présentant des anticorps avant ou après chirurgie avec des taux variants de $17 \%$ [84] à $32 \%$ [39]. Il semble toutefois qu'il n'y ait pas de différence lorsque l'on compare une population infertile avec ou sans varicocèle [78]. Après chirurgie, l'amélioration des paramètres spermatiques, notamment la mobilité, serait indépendante de la présence des anticorps [60].

Faute d'études, on ne connaît pas les effets à long terme des traumatismes testiculaires sur la fonction reproductive et la production d'anticorps. Il est possible que des traumatismes mineurs et/ou répétés (sports) puissent être suffisants pour entraîner une rupture de l'architecture testiculaire et la formation d'ASA. L'étude la plus récente portant sur 15 patients montre une perturbation des paramètres 
spermatiques plus en relation avec l'atrophie testiculaire qu'avec la présence d'anticorps [63].

La survenue d'une orchite auto-immune n'a jamais réellement été prouvée chez l'homme mais mise en évidence expérimentalement chez l'animal. Les orchites ourliennes n'entraînent qu'une augmentation sérique passagère des ASA [58].

Les troubles de l'éjaculation que l'on peut assimiler à des obstructions physiologiques généreraient la présence d'anticorps circulants et séminaux. On décrit des ASA dans les anéjaculations psychogènes [3] et chez les blessés médullaires. Les études sur les blessés médullaires sont contradictoires, que le sperme soit obtenu par vibromassage ou electroéjaculation [46, 91]. La qualité du sperme n'est pas imputable uniquement à la présence d'anticorps mais probablement aussi à l'infection fréquemment associée.

Dans le cancer du testicule, Guazzieri et coll. trouvent des taux d'autant plus élevés que la tumeur est à un stade avancé, $50 \%$ versus $30 \%$, avant même l'orchidectomie [40]. Une étude plus récente, mais après castration ou non descente testiculaire, décrit $18 \%$ d'ASA dans le sérum [51].

L'infection est le facteur le plus souvent retrouvé dans les antécédents du couple infertile. Les infections et/ou inflammations du tractus génital, sont dans de nombreuses études associées à une immunisation, et des taux élevés d'ASA sont retrouvés dans les orchites, épididymites aiguës, prostatites et urétrites [55, 72, 102]. Parmi les maladies sexuellement transmissibles, l'infection à chlamydiae est la plus souvent citée : une forte corrélation existe entre ASA et anticorps anti-chlamydiae de type IgA dans le sperme [101, 108] Eggert-Kruse et coll. n'ont pas, en 1998, retrouvé une incidence supérieure d'anticorps séminaux chez les couples présentant une infection asymptomatique [31]. Ce ne sont pas les anomalies spermatiques entraînées par l'infection chez l'homme qui sont la conséquence la plus grave pour Vigil et coll., en 2002, mais la transmission à la partenaire avec possibilité d'apparition d'anticorps anti-spermatozoïdes chez la femme [101].

Cette recherche d'anticorps doit être envisagée dans les stérilités inexpliquées de longue durée sans antécédents et sans autres raisons explicites [72].

En dépit de ces risques connus, très souvent de nombreux patients porteurs d'anticorps n'ont pas d'antécédents pathologiques retrouvés ni d'anomalies à l'examen génital : $50 \%$ dans une étude que nous avions réalisée, $43 \%$ dans l'étude de Hinting et coll. [45]. Une prédisposition génétique a été quelquefois évoquée. Dans $10 \%$ des cas, des anticorps antithyroïdiens sont retrouvés chez l'homme pouvant expliquer cette tendance génétique à l'auto-immunité $[5,85]$.

\section{Signes d'appel biologiques}

a) Les anomalies du spermogramme :

Souvent une auto-agglutination spontanée des spermatozoïdes incite à les rechercher. L'agglutination a lieu quand au moins 2 sites actifs se fixent sur au moins sur 2 cellules séparées. Selon la localisation de l'antigène sur le gamète, l'agglutination peut se faire par la tête, par le flagelle, ou impliquer toute la cellule : tête-tête, tête-flagelle, flagelleflagelle. Mais l'absence d'agglutinat n'est aucunement une preuve de l'absence d'ASA. Une asthénozoospermie primaire ou secondaire avec présence de spermatozoïdes mobiles lents ou sur place est aussi fréquemment rencontrée associée à une anomalie des paramètres du mouvement : vélocités, linéarité et amplitude du débattement de la tête quand les immunobilles sont positives [13, 27, 110, $111]$.

La vitalité peut être diminuée en relation avec la présence d'anticorps cytotoxiques. On peut noter aussi quelquefois une diminution de la numération des gamètes et une leucospermie. Un Mar test fait systématiquement en routine peut permettre de dépister ces anticorps, avec un taux de $5,4 \%$ dans notre étude [8]. Dans $80 \%$ des cas où le test aux immunobilles est supérieur à $10 \%$, il existe des ASA [24, 26]. Il faut toutefois préciser qu'à part quelques exceptions, la plupart des études épidémiologiques ne trouvent pas de différences significatives dans les principaux paramètres spermatiques entre les groupes d'hommes infertiles avec ou sans anticorps [36].

\section{b) Les tests dans la glaire :}

Ils représentent l'effet le mieux connu et le plus visible de l'interférence des anticorps avec la fertilité in vivo ou in vitro $[25,29,30,75]$. Les tests post-coitaux ou les tests de pénétration croisée sont négatifs ou pauvres en présence d'un mucus cervical ovulatoire. Cette altération de la pénétration des spermatozoïdes dans le mucus cervical est fonction du nombre et du type d'anticorps fixés à la surface du gamète (IgG ou IgA) [30,61]. C'est Jager en 1981 qui a suggéré le premier que l'interaction entre la partie Fc des IgG et des IgA serait responsable des mouvements d'oscillation sur place des spermatozoïdes, qu'il appelle "shaking phenomenon", et/ou de la non-pénétration dans le mucus [54]. Cette pénétration est corrélée négativement avec le taux d'immunoglobulines de type A [61].

\section{c) Echecs de fécondation ou pauci-fécondation en FIV classique :}

Ces échecs sont largement décrits. En présence de plus de $70 \%$, ou de $90 \%$ d'anticorps (selon les auteurs), IgG ou IgA, il est conseillé de proposer d'emblée une ICSI, surtout si les ASA sont situés sur la tête du gamète [1, 26, 36, $72,88,99]$. 


\section{INDICATIONS DE LA RECHERCHE DES ASA CHEZ LA FEMME}

Les indications ne paraissent pas aussi précises que chez l'homme. La pathogenèse de cette immunisation est encore moins claire. Alors que pendant la période d'activité sexuelle, le tractus féminin est inséminé de façon répétée, seule une minorité de femmes développe des anticorps [70].

\section{Signes d'appel cliniques}

Les infections pelviennes sont reconnues responsables d'une incidence importante d'anticorps. Comme chez l'homme, l'apparition des anticorps est consécutive à un phénomène inflammatoire et/ou à une effraction de la muqueuse [101, 102]. Sont incriminés surtout les chlamydiae et mycoplasmes (32). Cunningham et coll. retrouvent $59 \%$ d'ASA dans les atteintes pelviennes hautes qui sont, pour ces auteurs, les plus immunogènes [20]. Les infections génitales pourraient jouer un rôle en altérant l'efficacité des facteurs immunosuppresseurs du sperme ou en conduisant à la formation d'anticorps réagissant de façon croisée avec les spermatozoïdes.

Les relations entre endométriose et anticorps sont discutées, notamment pour les lésions les moins sévères ; selon Leach et coll., la proportion n'est pas significativement différente entre les femmes infertiles et celles qui présentent une endométriose [64]. D'autres études démontrent le contraire en comparant le sérum et le fluide folliculaire. Le taux d'anticorps est plus élevé dans le fluide péritonéal que dans le sérum, cela à la fois chez les femmes infertiles avec endométriose et dans les infertilités idiopathiques. Ceci ne prouve pas que l'endométriose en elle-même soit un facteur prédisposant à l'apparition des anticorps antispermatozoïdes, mais la production locale est significativement importante [52, 81, 95]. Dans toutes ces études, on retrouve l'association avec la présence d'auto-anticorps (ex : anti-zona) $[52,81,95,96]$.

L'implication des ASA dans les fausses couches à répétition n'est pas confirmée dans les études récentes, ce qui est en contradiction avec les résultats préliminaires de Witkin et coll. en 1989 [21, 105, 106]. Il est important que ce facteur soit réévalué ; toutefois, on retrouve là aussi une proportion plus élevée d'auto-anticorps chez ces femmes [2].

La ménopause précoce pourrait être responsable d'immunisation car le déficit oestrogénique est responsable des modifications des lymphoctes $\mathrm{T}$, modifications qui sont normalisées après deux ans de traitement substitutif oestrogénique [50].

Les interventions au niveau du col utérin pour néoplasie intra-épithéliale sont rendues responsables d'immunisation, sans doute par contact avec les spermatozoïdes trop tôt après la chirurgie [80].
Au contraire des résultats retrouvés chez les homosexuels, les pratiques sexuelles et les partenaires multiples ne semblent pas être un facteur de risque chez la femme [12]. Durant l'activité sexuelle, les muqueuses sont régulièrement en contact avec les spermatozoïdes et il n'est pas retrouvé d'anticorps. Une étude américaine sur des femmes ayant été inséminées par de multiples donneurs, jusqu'à 44 cycles, ne retrouve pas d'anticorps [70]. Une étude chez des prostituées a retrouvé $43 \%$ d'ASA mais avec des problèmes infectieux associés [4].

Les inséminations intra-utérines ou intra-péritonéales n'augmentent pas l'incidence des anticorps, même si une grande quantité des spermatozoïdes est phagocytée dans la cavité péritonéale $[38,69]$. Il n'est mis en évidence qu'une production transitoire d'anticorps sériques à des taux bas.

La difficulté de toutes ces études réside dans les comparaisons de populations et la méthodologie employée, et même si Stern et coll. trouvent une corrélation entre les taux d'anticorps dans les différents compartiments, la présence fréquente d'anticorps à un titre faible dans le sérum semble avoir une signification clinique contestable car l'incidence est identique chez les femmes fertiles [94].

\section{Signes d'appel biologiques}

a) Tests dans la glaire :

La présence d'anticorps pourra être suspectée face à de tests post-coïtaux négatifs ou déficients avec non-pénétration ou surtout immobilisation des gamètes $[10,14,30]$. Le test croisé in vitro confirme ces résultats en présence de sperme et glaire témoins. L'immobilisation des spermatozoïdes peut aussi être une conséquence des anticorps fixant le complément si les facteurs anti-complémentaires sont en proportion trop faible dans la glaire et le plasma séminal.

\section{b) Echecs de PMA inexpliqués :}

La présence d'anticorps dans le sérum et surtout dans le fluide folliculaire incite certains auteurs à préconiser la FIV ou l'ICSI [1, 26, 36, 72, 88, 99].

\section{CONCLUSION}

La recherche des facteurs prédisposants ne doit pas être oubliée dans l'interrogatoire du couple infertile, même si ces antécédents ne sont peut-être qu'une potentialisation possible du risque d'apparition d'anticorps. La relation entre infertilité et anticorps est reconnue mais contestée ; c'est une question que l'on ne peut résoudre facilement, et des études prospectives plus larges sont encore nécessaires [44, 49]. Une publication en 1998 de Culligan et coll. affirme que les tests sont peu prédictifs des échecs de fécondation, donc inutiles [19]. Comme il n'existe pas en effet de réels 
traitements hors des procréations médicalement assistées, cette recherche n'entraînerait-t-elle pas une majoration du coût de la prise en charge [10] ?

A l'heure de l'ICSI, quelle est la place de la recherche des anticorps en pratique clinique ? Je cite Helmerhorst et coll. en 1999 : "At the moment it is difficult to consider the routinely used antisperm antibody (ASA) tests as an essential procedure in the fertility work-up nor that it is even more difficult to justify a treatment on the basis of such tests" [44]. Voici bien un exemple du "nihilisme thérapeutique" dénoncé par Bronson en 1999 [11].

La conséquence du succès de l'ICSI dans cette indication est la diminution de la recherche sur la compréhension de l'origine de ces anticorps $[15,17,77]$. Or, il est toujours plus satisfaisant d'avoir un diagnostic exact, une explication probable de l'infertilité du couple. En effet, cela peut permettre :

- la progression de la connaissance sur l'histoire naturelle de l'apparition des anticorps et des interactions anticorps-antigènes, sur les effets précis d'interaction avec le processus de fécondation et peut-être une éventuelle possibilité de prévention ;

- l'identification des antigènes impliqués dans ce processus pour permettre de pouvoir ainsi développer des tests ELISA plus spécifiques ;

- une stratégie pour une prise en charge plus rapide du couple à l'aide à la procréation médicalement assistée la plus adaptée et la moins pénible, en fonction du type et de la localisation des ASA ainsi que de leur taux ;

- enfin, ces informations pourraient nous permettre d'avancer dans le développement de méthodes immunologiques de contraception.

\section{REFERENCES}

1. ABSHAGEN K., HERMANN M.D., TREVOR M.D. et al. : Influence of sperm surface antibodies on spontaneous pregnancy rate. Fertil. Steril., 1998, $70: 355-356$.

2. AL-HUSSEIN K., AL-MUKHALAFI Z., BERTILSSON P.A. et al. : Value of flow cytometric assay for the detection of antisperm antibodies in women with an history of recurrent abortion. Am. J. Reprod. Immunol., 2002, 47 : 31-37.

3. ALMAGOR M., DAN-GOOR M., HOVAV Y. et al. : Antisperm antibodies in men with psychogenic ejaculation. Arch. Androl., 1998, $41: 1-4$.

4. BAHRAMINEJAD R., KADANALI S., ERTEN O. et al. : Reproductive failure and antisperm antibodies production among prostitutes. Acta Obstet. Gynecol. Scand., 1991, 70 : 483-485.

5. BAKER H.W.G., CLARKE G.N., McGOVAN M.P. et al. :
Increased frequency of autoantibodies in men with sperm antibodies. Fertil. Steril., 1985, $43: 438-441$.

6. BANDOH R., YAMANO S., KAMADA M. et al. : Effect of immobilizing antibodies on the acrosome reaction of human spermatozoa. Fertil. Steril., 1992, 57: 387-392.

7. BARRAT C.L., HARRISON P.E., ROBINSON A. et al. : Antisperm antibodies and lymphocytes subsets in semen, not a simple relationship. Int. J. Androl., 1990, $13: 50-58$.

8. BARTHELEMY C., LECOMTE P., LECOMTE C. et al. : Utilisation du Mar test au latex pour le dépistage en routine des anticorps agglutinants antispermatozoïdes chez les sujets présentants une autoagglutination spontanée. J. Gynécol. Obstet. Biol. Reprod., 1986, 15 : 287-291.

9. BRONSON R.A., O'CONNOR W.J., WILSON T.A. et al. : Correlation between puberty and the development of autoimmunity to spermatozoa in men with cystic fibrosis. Fertil. Steril.,1992, $58: 1199-1204$.

10. BRONSON R.A. : Antisperm antibodies : a critical evaluation and clinical guidelines. J. Reprod. Immunol., 1999, 45 : 159-183.

11. BRONSON R.A. : Detection of sperm antibodies : an argument against a therapeutic nihilism. Hum. Reprod., 1999, 14 : 16711673.

12. CHACHO K.J., HAGE C.W., SHULMAN S. : The relationship between female sexual practices and the development of antisperm antibodies. Fertil. Steril., 1991, $56: 461-464$.

13. CHECK J.H., ADELSON H.J., BOLLENDORF A. : Effect of antisperm antibodies on computerized semen analysis. Arch. Androl., 1991, 27 : 61-63.

14. CHECK J.H., BOLLENDORF A., KATSOFF D. et al. : The frequency of antisperm antibodies in cervical mucus of women with poor post-coital tests and their effect on pregnancy rates. Am. J. Reprod. Immunol., 1994, 32 : 38-42.

15. CHECK M.L., CHECK J.H., KATSOFF D. et al. : ICSI as an effective therapy for male factor with antisperm antibodies. Arch. Androl., 2000, 45 : 125-130.

16. CLARKE G.N. : Immunoglobulin class and regional specificity of antispermatozoal antibodies blocking cervical mucus penetration by human spermatozoa. Am. J. Reprod. Immunol., 1988, 16: 135-138.

17. CLARKE G.N., BOURNE H., BAKER H.W. : Intracytoplasmic sperm injection for treating infertility associated with sperm autoimmunity. Fertil .Steril., 1997, 68 : 112-117.

18. COLLINS J.A., BURROWS E.A., YEO J. et al. : Frequency and predictive value of antisperm antibodies among infertile couples. Hum. Reprod,. 1993, 8 : 592-598.

19. CULLIGAN P.J., CRANE M.M., BOONE W.R. et al. : Validity and cost-effectiveness of antisperm antibody testing before in vitro fertilization. Fertil. Steril., 1998, 69 : 894-898.

20. CUNNINGHAM D.S., FULGHAM D.L., RAY D.L. : Antisperm antibodies to sperm surface antigens in women with genital tract infections. Am. J. Obstet. Gynecol., 1991, 164 : 791-796.

21. DAYA S. : Issues in the etiology of recurrent spontaneous abortion. Curr. Opin. Obstet. Gynecol., 1994, 6 : 153-159.

22. D'CRUZ O.J., HAAS JR G.G. : Lack of complement activation 
in the seminal plasma of men with antisperm antibodies associated in vivo on their sperm. Am. J. Reprod. Immunol., 1990, 24 : 51-57.

23. D'CRUZ O.J., HAAS G.G., DE LA ROCHA R. et al. : Occurrence of serum antisperm antibodies in patients with cystic fibrosis. Fertil. Steril., 1991, $56: 519-527$.

24. DE ALMEIDA M., SOUMAH A., DUCOT B. et al. : Dans quelles circonstances cliniques doit-on évoquer une stérilité masculine immunologique ? Les méthodes de détection des anticorps circulants. Contracep. Fertil. Sex., 1983, 11 : 353-356.

25. DE ALMEIDA M., SOUMAH A., JOUANNET P. : Incidence of sperm associated immunoglobulins in infertile men with suspected autoimmunity to sperm. Int. J. Androl., 1986, $9: 321-330$.

26. DE ALMEIDA M. : Immunologie et hypofertilités masculines. Rev. Fr. Gynécol. Obstet., 1989, 84 : 112-118.

27. DE ALMEIDA M., ZOUARI P., JOUANNET P. et al. : In vitro effects of antisperm antibodies on human sperm movement. Hum. Reprod., 1991, $6: 405-410$.

28. DE KRETSER D.M., HUIDOBRO C., SOUTHWICK G.J. : The role of epididymis in human infertility. J. Reprod. Fertil.,1998, 53, Suppl. : 271-275.

29. EGGERT-KRUSE W., HOFSAB A., HAURY E. et al. : Relationship between local antisperm antibodies and sperm-mucus interaction in vitro and in vivo. Hum. Reprod., 1991, 6 : 267-276.

30. EGGERT-KRUSE W., BOCKEM-HELLWIG S., DOLL A. et al.: Antisperm antibodies in cervical mucus in an unselected subfertile population. Hum. Reprod., 1993, 8 : 1025-1031.

31. EGGERT-KRUSE W., BUHLINGER-GOPFARTH N., ROHR G. et al : Antibodies to Chlamydiae trachomatis in semen and relationship with parameters of male fertility. Hum. Reprod., 1996, $11: 1408-1417$.

32. EGGERT-KRUSE W., ROHR G., PROBST S. et al. : Antisperm antibodies and microorganisms in genital secretions : a clinically signifiant relationship ? Andrologia, 1998, 30 suppl. $1: 61-71$.

33. FILIPPINI A., RICCIOLI A., PADULA F., et al : Immunology and immunopathology of the male genital tract : Control and impairment of immune privilege in the testis and semen. Hum. Reprod. Update, 2001, $7: 444-449$.

34. FLICKINGER C.J., BARAN M.L., HOWARDS S.S. : Epididymal obstruction during development results in antisperm antibodies at puberty in rats. J. Androl., 1998, 19 : 2136-2144.

35. FRANCAVILLA F., ROMANO R., SANTUCCI R. et al : Interference of antisperm antibodies with the induction of the acrosome reaction by zona pellucida (ZP) and its relationship with the inhibition of zona binding. Fertil. Steril., 1997, 67 : 1128-1133.

36. FRANCAVILLA F., ROMANO R., SANTUCCI R. et al : Naturally occuring antisperm antibodies in men : interference with fertility and implications for treatment. Front. Biosc., 1999, 4 : 925.

37. FRASIER I., SLATER N., TATE C. et al. : Testicular torsion does not cause autoimmunisation in man. Br. J. Surg., 1985, 72 : 237-238.

38. FRIEDMAN A.J., JUNEAU N.M., SEDENSKY B. : Antisperm antibody production following intra-uterine insemination. Hum.
Reprod., 1991, $6: 1125-1128$.

39. GILBERT B.R., WITKIN S.S., GOLDSTEIN M. : Correlation of sperm bound immunoglobulins with impaired sperm analysis in infertile men with varicocoele. Fertil. Steril., 1989, $52: 469$ 473.

40. GUAZZERI S., LEMBO A., FERRO G. et al. : Sperm antibodies and infertility in patients with testicular cancer. Urology, 1985, $26: 139-142$.

41. HARRISON S., ULL G., PILLAI S. : Sperm acrosome status and sperm antibodies in infertility. J. Urol., 1998, $159: 1554-$ 1558.

42. HEDGER M.P. : Macrophages and the immune responsivness of the testis. J. Reprod. Immunol., 2002, $57: 19-34$.

43. HEIDENREICH A., BONFIG R., WILBERT D.M., et al : Risk factors for antisperm antibodies in infertile men. Am. J. Reprod. Immunol., 1994, $31:$ 69-76.

44. HELMERHORST F.M., FINKEN M.J.J., ERWICH J.J. : Detection assays for antisperm antibodies : what do they test ? Hum. Reprod., 1999, 14 : 1669-1670.

45. HINTING A., SOEBADI D.M., SANTOSO R.I. : Evaluation of the immunological cause of male infertility. Andrologia, 28, 1996 : 123-126.

46. HIRSCH I.H., SEDOR J., CALLAHAN H. J, et al. : Antisperm antibodies in seminal plasma in spinal cord injuries men. Urology, 1992, $39: 243-247$.

47. HJORT T., HUSTED S., LINNET-JEPSEN P. : The effect of testis biopsy on autosensitization against spermatozoal antigens. Clin. Exp. Immunol., 1974, 18 : 201-212.

48. HJORT T. : Do antibodies to sperm reduce fecundity ? A mini review in historical perspective. Am. J. Reprod. Immunol., 1998, $40: 215-222$.

49. HJORT T. : Antisperm antibodies and infertility : an unsolvable question ? Hum. Reprod., 1999, 14 : 2423-2426.

50. HO P.C., TANG G.W., LAWTON J.W. : Lymphocytes subsets and serum immunoglobulins in patients with premature ovarian failure before and after oestrogen replacement. Hum. Reprod., 1993, $8: 714-716$.

51. HOBARTH K., KLINGER H.C., MAIER U., et al. : Incidence of sperm antibodies in patients with carcinoma of the testis and in subfertile men with normogonadotrophic oligoasthenozoospermia. Urol. Int., 1994, 52 : 162-165.

52. IBORRA A., PALACIO J.R., ULCOVA-GALLOVA Z. et al. : Autoimmune response in women with endometriosis. Am. J. Reprod. Immunol., 2000, 44 : 236-241.

53. IMADE G.E., BAKER H.W., DE KRETSER D.M. : Immunosuppresive activities in the seminal plasma of infertile men : relationship to sperm antibodies and autoimmunity. Hum. Reprod., 1997, 12 : 56-262.

54. JAGER S., KREMER J., KUIKEN J. et al. : The signifiance of the Fc part of antispermatozoal antibodies for the shaking phenomenon in the sperm-cervical mucus contact test. Fertil. Steril., $1981,36: 792$.

55. JAROW J.P., KIRKLAND J.A., ASSIMOS D.J. : Association of antisperm antibodies with chronic non bacterial prostatitis. Urology, 1990, $36: 154-156$. 
56. JAROW J.P., SANZONE J.J. : Risk factors for male partners antisperm antibodies. J. Urol., 1992, 148 : 1805-1807.

57. JAROW J.P. GOBULOFF E.T., CHANG T.S. et al. : Relationship between antisperm antibodies and testicular histologic changes in humans after vasectomy. Urology. 1994, 43 : 521-524.

58. KALAYDJIEV S., DIMITROVA D., NENOVA M. et al. : Serum sperm antibodies are not elevated after mumps orchitis. Fertil. Steril., 2002, $77:$ 76-82.

59. KAY D.J., CLIFTON V., TAYLOR J.S. et al : Antisperm antibodies and semen profile in re-anastomosed men. Reprod. Fertil. Dev., 1993, 5 : 135-139.

60. KNUDSON G., ROSS L., STUHLDREHER D. et al. : Prevalence of sperm bound antibodies in infertile men with varicocoele : the effect of varicocoele ligation on antibodies levels and semen response. J. Urol., 1994, 151 : 1260-1262.

61. KREMER J., JAGER S. : The signifiance of antisperm antibodies for sperm-cervical mucus interaction. Hum. Reprod., 1992, 7 : 781-784.

62. KOSAR A., KUPELI B., ALCIGIR G. et al. : Immunologic aspect of testicular torsion : detection of antisperm antibodies in controlateral testis. Eur. Urol., 1999, 36 : 640-644.

63. KUKADIA A.N., ERCOLE C.J., GLEICH P. et al. : Testicular trauma : potential impact on reproductive function. J. Urol., 1996, 156 : 1643-1646.

64. LEACH R.E., ARNESON B.W., BALL G.D. : Absence of sperm antibodies and factors influencing sperm motility in the cul-desac fluid of women with endometriosis. Fertil. Steril., 1990, 53 : 351-353.

65. LIU D.Y., CLARKE G.N., BAKER H.W. : Inhibition of human sperm zona pellucida and sperm-oolemma binding by antisperm antibodies. Fertil. Steril., 1991, 55 : 440-442.

66. LIU J., ZHANG Y., SHEN G. et al. : An approach to pathogenesis of male infertility with antisperm antibodies. Int. J. Fertil., 1993, $38: 187-191$.

67. LOMBARDO F., GANDINI L., DONDERO F. et al : Immunology and immunopathology of the male genital tract : Antisperm immunity in natural and assisted reproduction. Hum. Reprod. Update, 2001, $7: 450-456$.

68. McLACHLAN R.I. : Basis, diagnosis and treatment of immunological infertility in men. J. Reprod. Immunol., 2002, 57 : 35-45.

69. MARSHBURN P.B., KUTTEH W.H. : The role of antisperm antibodies in infertility. Fertil. Steril., 1994, 61 : 799-811.

70. MASHU A., TANABE K., KAMEI K. et al. : Sperm immobilizing antibodies in women undergoing artificial insemination with donors sperm. Fertil. Steril., 1994, 62 : 651-653.

71. MATSUDA T., MUGURUMA K., HORII Y. et al. : Serum antisperm antibodies in men with vas deferens obstruction caused by childhood inguinal herniorrhaphy. Fertil. Steril.,1993, 59 : 10951097.

72. MAZDUMAR S., LEVINE A.S. : Antisperm antibodies : etiology, pathogenesis, diagnosis and treatment. Fertil. Steril., 1998, $70: 799-810$.

73. MEINERTZ H., LINNET L., FOGH ANDERSON P. : Antisperm antibodies and fertility after vasovasostomy : a follow-up study of 216 men. Fertil. Steril., 1990, $54: 315-321$.
74. MEINERTZ H., LINNET L., WOLF H. et al. : Antisperm antibodies on epididymal spermatozoa. Am. J. Reprod. Immunol., 1991, $25: 158-162$.

75. MENGE A.C., MEDLEY N.E., MANGIONE C.M. : The incidence and influence of antisperm antibodies in infertile human couples on sperm-cervical mucus interactions and subsequent fertility. Fertil. Steril., 1982, $38: 439-446$.

76. MIRILAS P., DE ALMEIDA M. : Absence of antisperm surface antibodies in prepuberal boys with cryporchidism and other anomalies of the inguinoscrotal region before and after surgery. $J$. Urol., 1999, $162: 177-181$.

77. NAGY Z.P., VERHEYEN G., TOURNAYE H. : Special application of intracytoplasmic sperm injection : the influence of sperm count, motility, morphology, source and sperm antibodies on the outcome. Hum. Reprod, 1998, 1 : 143-154.

78. NAUGHTON C.K., NANGIA A.K., AGARWAL A. : Varicocoele and male infertility : Part II Pathophysiology of varicocoele in male fertility. Hum. Reprod. Update, 2001, 7 : 473-481.

79. NAZ R.K. : Effects of antisperm antibodies on early cleavage of fertilized ova. Biol. Reprod., 1992, 46 : 130-139.

80. NICHOLSON S.C., ROBINSON J.N., SARGENT I.L. et al. : Does large loop excision of the transformation zone of the cervix predispose to the development of antisperm antibodies in women ? Fertil. Steril., 1996, $65: 871-873$.

81. NIP M.M., TAYLOR P.V., RUTHERFORD A.J. et al. : Autoantibodies and antisperm antibodies in sera and follicular fluids of infertile patients; relation to reproductive outcome after in vitro fertilization. Hum. Reprod., 1995, 10 : 2564-2569.

82. NISTAL M., RIESTRA M.L., PANIGUA R. : Focal orchitis in undescended testes : discussion of pathogenic mechanisms of tubular atrophy. Arch. Pathol. Lab. Med., 2002, 126 : 64-69.

83. OHL D., NAZ R.K. : Infertility due to sperm antibodies. Urology, 1995, $46: 591-602$.

84. OSHINSKY G.S., RODRIGUEZ M.W., MELLINGER B.C. : Varicocele-related infertility is not associated with increased sperm bound antibodies. J. Urol.,1993, 150: 871-873.

85. PASCHKE R., SCHULZE BERTELSBECK D., TSALIMALMA K. et al. : Association of sperm antibodies with other antibodies in infertile men. Am. J. Reprod. Immunol.,1994, 32 : 88-94.

86. PATRIZIO P., SILBER S.J., ORD T. et al. : Relationship of epidiymal sperm antibodies to their in vitro fertilization capacity in men with congenital absence of the vas deferens. Fertil. Steril., 1992, $58: 1006-1010$.

87. PRICE R.J., ROBERT T.K., GREEN D. et al. : Anticomplementary activity in human semen and its possible importance in reproduction. Am. J. Reprod. Immunol., 1984, 6 : 92-98.

88. RAJAH S.V., PARSLOW J.M., HOWELL R.J. et al. : The effects on in vitro fertilization of antibodies to spermatozoa in subfertile men. Hum. Reprod., 1993, 8 : 1079-1082.

89. RÜMKE P. : The presence of antisperm antibodies in serum of two patients with oligospermia. Vox. Sang., 1954, $4: 135-140$.

90. SINISI A.A., D'APUZZO A., PASQUALI D. et al. : Antisperm antibodies in prepubertal boys treated with chemotherapy for malignant or non malignant diseases and in boys with genital abnormalities. Int. J. Androl., 1997, 20 : 23-28. 
91. SIOSTEEN A., SEEN Y., FORSSMAN L. et al. : Autoimmunity to spermatozoa and quality of semen in men with spinal cord injuries. Int. J. Fertil., 1993, $38: 117-122$.

92. SNELL W.J., WHITE J.M. : The molecules of mammalian fertilization. Cell, 1996, 85 : 629-637.

93. STEELE E.K., ELLIS P.K., LEWIS S.E.M. et al : Ultrasound, antisperm antibody, and hormone profiles after testicular trucut biopsy. Fertil. Steril., 2001, 75 : 423-428.

94. STERN J.E., DIXON P.M., MANGANIELLO P.D. et al. : Antisperm antibodies in women : variability in antibody level in serum, mucus and peritoneal fluid. Fertil. Steril., 1992, 58 : 950958.

95. SZCZEPANSKA M., SKZYPCZAK J., KAMIENICZNA M. et al. : Antizona and antisperm antibodies in women with endometriosis and/or infertility. Fertil. Steril., 2001, $75: 97-105$.

96. ULCOVA-GALLOVA Z., BOUSE V., SVABEK L. et al. : Endometriosis in reproductive immunology. Am. J. Reprod. Immunol., 2002, $47: 269-274$.

97. URRY R.L., CARRELL D.T., STARR N.T. et al. : The incidence of antisperm antibodies and infertility in patients with a history of cryporchidism. J. Urol., 1994, $151: 381-383$.

98. VASQUEZ-LEVIN M., KAPLAN P.,GUZMAN I. et al. : The effect of female antisperm antibodies on the in vitro fertilization, early embryonic development and pregnancy outcome. Fertil. Steril., 1991, $56: 84-88$.

99. VASQUEZ-LEVIN M., NOTRICA J.A., POLAK DE FRIED E.: Male immunologic infertility : sperm performance on in vitro fertilization. Fertil. Steril., 1997, $68: 675-681$.

100. VERDIER Y., CHAUFFAUX S., BOUE F. : Identification of post vasectomy sperm auto-antigens in fox (Vulpes vulpes) by two dimensionnal gel electrophoresis and western blotting. J. Reprod. Immunol., 2002, 54 : 65-80.

101. VIGIL P., MORALES P., TAPIA A. et al. : Chlamydiae trachomatis infection in male partners of infertile couples : Incidence and sperm function. Andrologia, 2002, 34 : 155-161.

102. WITKIN S.S., TOTH A. : Relationship between genital tract infections, sperm antibodies in seminal fluid and infertility. Fertil. Steril., 1983, $40: 805-808$.

103. WITKIN S.S. : Mechanisms of active suppression of the immune response to spermatozoa. Am. J. Reprod. Immunol. Microbiol., 1988, 7 : 61-64.

104. WITKIN S.S., DAVID S.S. : Effects of antisperm antibodies on pregnancy outcome in a subfertile population. Am. J. Obstet. Gynecol., 1988, $158: 59-62$.

105. WITKIN S.S., CHAUDHRY A. : Relationship between circulating antisperm antibodies in women and autoantibodies on the ejaculated sperm of their partners. Am. J. Obstet. Gynecol., 1989, $161: 900-903$.

106. WITKIN S.S., CHAUDHRY A. : Association between recurrent spontaneous abortions and circulating IgG antibodies to sperm tail in women. J. Reprod. Immunol., 1989, 15 : 151-158.

107. WITKIN S.S., VITI D., DAVID S.S. et al. : Relation between antisperm antibodies and the rate of fertilization of human oocytes in vitro. J. Assist. Reprod. Genet., 1992, 9 : 9-13.
108. WITKIN S.S., VITI D., DAVID S.S. et al. : Relationship between asymptomatic male genital tract exposure to Chlamydiae trachomatis and autoimmune response to spermatozoa. Hum. Reprod., 1995,10 : 2952-2955.

109. YANAGIMACHI R., OKADA A., TUNG K.S.K. : Sperm auto antigens and fertilization. II Effects of anti-guinea pig sperm autoantibodies on sperm ovum interactions. Biol. Reprod., $1981,24: 512-518$

110. ZOUARI R., DE ALMEIDA M., FENEUX D. et al. : Effects of sperm associated antibodies on the dynamics of sperm movement and on the acrosome reaction of human spermatozoa. J. Reprod. Immunol. 1992, 22 : 59-72.

111. ZOUARI R., DE ALMEIDA M., RODRIGUEZ D. : Localisation of antibodies on spermatozoa and sperm movement characteristics are good predictors of in vitro fertilization success in case of male autoimmune infertility. Fertil. Steril., 1993, 59 : 606-612.

\author{
ABSTRACT \\ Indications for antisperm antibody testing
}

Claire BARTHELEMY

The clinical significance of antisperm antibodies (ASA) is highly controversial. A significant percentage of infertile men and women present immunity to spermatozoa, suggesting that ASA may interfere with the fertilizing capacity. ASA can act negatively on sperm parameters, sperm-cervical mucus interaction, gamete fusion and possibly also on the first step of embryonic development. ASA are present in approximately $2.8 \%$ to $26 \%$ of the male population and $0.2 \%$ to $1.6 \%$ of women. The pathogenesis of immunity to spermatozoa had not been fully elucidated: breakdown of normal protective mechanisms, i.e. blood-testis barrier, or epithelial barrier in women, and other mechanisms of immunological sperm tolerance, such as regulation of suppressor T lymphocytes.

The indication for antisperm antibody testing is based on clinical and laboratory findings of infertile patients. In men, indictions for ASA testing include a history of genital disease, surgery for genital abnormalities, vasectomy, obstruction or injuries of the male genital tract, infection of accessory glands, long-standing infertility, alteration of semen parameters (agglutination, motility), mucus penetration, and reduced fertilizing capacity in IVF. In many 
cases, no etiological cause of autoimmunity is found and a genetic predisposition has been suggested. A majority of women do not develop antisperm antibodies, despite repeated contact with spermatozoa during their sexual life. Upper genital tract infection is the main cause of isoimmunization in females, although sexual practices, endometriosis, surgery for cervical neoplasia, recurrent spontaneous abortion and long-term infertility may also be involved. Sperm-cervical mucus impairment is the most obvious effect of immunization in women associated with IVF failure. Autoantibodies are frequently associated with antisperm antibodies.

One of the consequences of the success of ICSI has been a decreased research effort to further the understanding of the origin and relevance of antisperm antibodies and specific antibody-antigen interactions. A better understanding of the natural history of immunological infertility would be useful for patient conseiling and to develop the most effective, efficient and safest management strategies. Such data could also be useful for the development of new tests and immunological methods of male contraception.

Key-words: antisperm antibodies, ASA, human sperm, immunological infertility, human 